\title{
Index and First-Generation Cases in a COVID-19 Outbreak — Jilin Province, China, 2021
}

\author{
Weihua Cheng, ${ }^{1, \alpha}$; Bing Zhao ${ }^{2, \alpha}$; Enfu Chen ${ }^{3,8}$; Guoqian $\mathrm{Li}^{1}$; Jun $\mathrm{Ma}^{1}$; Yanling Cui'; \\ Changxi $\mathrm{Xu}^{4}$; Yong Cui ${ }^{4}$; Bo Shen ${ }^{4}$; Mingyu Luo ${ }^{3}$; Dapeng Yin ${ }^{2, *}$; Laishun Yao ${ }^{4, *}$
}

\section{Summary}

What is already known on this topic?

Contact tracing and testing with isolated medical care of identified cases is a key strategy for interrupting chains of transmission of COVID-19 and reducing mortality associated with COVID-19. At the early phases of the COVID-19 pandemic, due to test capacity limitations, case finding often started from suspected cases.

\section{What is added by this report?}

The index patient infected 74 individuals who were close contacts that were identified through contact tracing, and exposed individuals were monitored in quarantine with daily polymerase chain reaction (PCR) testing. All individuals were asymptomatic initially, but all PCR-positive individuals eventually developed symptoms. Infectivity was documented up to 8 days before being confirmed as a symptomatic case, approximately 4 days before turning PCR positive.

What are the implications for public health practice?

During an outbreak, we suggest tracing close contacts from both PCR-positive individuals and suspected cases, rather than from suspected cases alone. Due to the long period of infectivity before turning PCR positive or developing symptoms, close contacts that had contact with a newly PCR positive case within 4 days should be judged as at risk of being infected; close contacts that had contact within 8 days of a newly symptomatic case should be judged as at risk being infected.

Contact tracing - along with robust testing, isolation, and care of cases - is a key strategy for interrupting chains of transmission of coronavirus disease 2019 (COVID-19), caused by COVID-19 virus also known as severe acute respiratory syndrome coronavirus 2 (SARS-CoV-2), and reducing mortality associated with COVID-19 (1-2). Close contacts are defined as individuals who have had contact, without effective protection, with one or more suspected or confirmed COVID-19 cases any time starting 2 days before onset of the suspected or confirmed cases' symptoms or 2 days before sampling for laboratory testing of asymptomatic infected persons (3-4). At the early phases of COVID-19 pandemic, the finding of COVID-19 cases usually began from suspected cases due to delayed testing capabilities (5).

On January 9, 2021, the first case (Patient A) of a COVID-19 outbreak arrived unknowingly infected in Dongchang District, Tonghua City, Jilin Province, China. He gave three product promotion lectures in Location $\mathrm{A}$ and infected audience members during the lectures. By January 31, 2021, 74 lecture participants were confirmed to have been infected and were considered first-generation cases. The Tonghua CDC led an investigation of this outbreak that ultimately identified 140 cases (G). In this article, we focused on the 74 first-generation cases, which all had known exposure times and locations. Once Patient A had been diagnosed using polymerase chain reaction (PCR) testing as being infected, lecture participants were located and placed in quarantine where they were tested with PCR and evaluated for COVID-19 symptoms every day. This careful observation period under quarantine provided an opportunity to assess infectivity period, incubation time, the time between becoming PCR positive and developing symptoms. We report results of our study of first-generation cases to further improve investigation and management of close contacts of COVID-19 cases.

\section{INVESTIGATION AND RESULTS}

We obtained data about the index case and firstgeneration cases from the National Infectious Diseases Reporting System (NIDRS) and the Tonghua CDC outbreak investigation. In response to the outbreak, close contacts were quarantined, and nucleic acid testing was conducted daily to determine whether the contacts had been infected. Contacts who turned PCR 
positive were transferred from quarantine to a hospital and were reported to NIDRS within 2 hours. CDC staff would complete an epidemiological report within 24 hours. As soon as PCR-positive individuals developed symptoms, they would be classified by physicians as confirmed cases, and their NIDRS record would be updated with their new status as a confirmed COVID-19 case.

On January 10 and 11, Patient A gave three lectures in Site A in Tonghua City. As of January 31, 74 close contacts were diagnosed as COVID-19 confirmed cases. All were first-generation PCR-positive cases, and all were PCR-positive, first-generation infected individuals ultimately became symptomatic. The median age was 72 (inter-quartile range, 60-87) years (Figure 1A); $12(16.22 \%)$ were critical cases, 10 $(13.51 \%)$ were severe cases, $47 \quad(63.51 \%)$ were moderate cases, and $5(6.76 \%)$ were mild cases.

All first-generation cases had attended lectures by Patient A on January 10 or 11 . The average time between exposure (lecture) and turning PCR-positive while in quarantine was 6.15 days (95\% CI: 5.65-6.65) (Figure 1B). The average time between exposure and being diagnosed as confirmed (symptomatic, PCR-
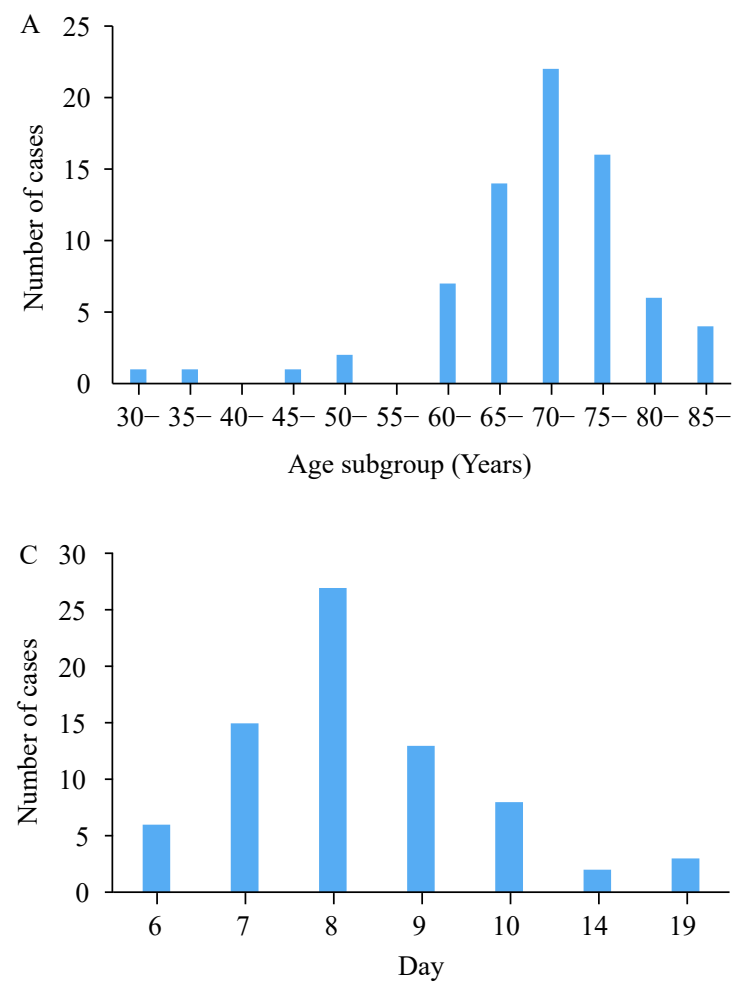

positive) cases was 8.64 days (95\%CI: 8.04-9.23) (Figure 1C). The average time between turning PCRpositive and being diagnosed as confirmed cases was 2.49 days (95\%CI: 2.00-2.92).

Patient $A$ had been infected by an adjacent passenger on train K350 on January 5, 2021 (7). He tested PCRpositive on January 12, 2021 and was diagnosed as a confirmed (symptomatic) case on January 16 . He infected participants who attended his lecture in Changchun City, Jilin Province on January 8. Therefore, the earliest date that Patient $A$ had documented infectivity was January 8, 4 days before Patient A tested PCR-positive and 8 days before his diagnosis as a confirmed case (Figure 2).

\section{DISCUSSION}

During the earliest phase of the COVID-19 pandemic in China, the finding of COVID-19 cases was often delayed due to insufficient laboratory capacity, with long times between development of symptoms (i.e., a suspected case) and being confirmed as COVID-19 cases by PCR (4). Markedly improved PCR testing capacity was instrumental in stopping the
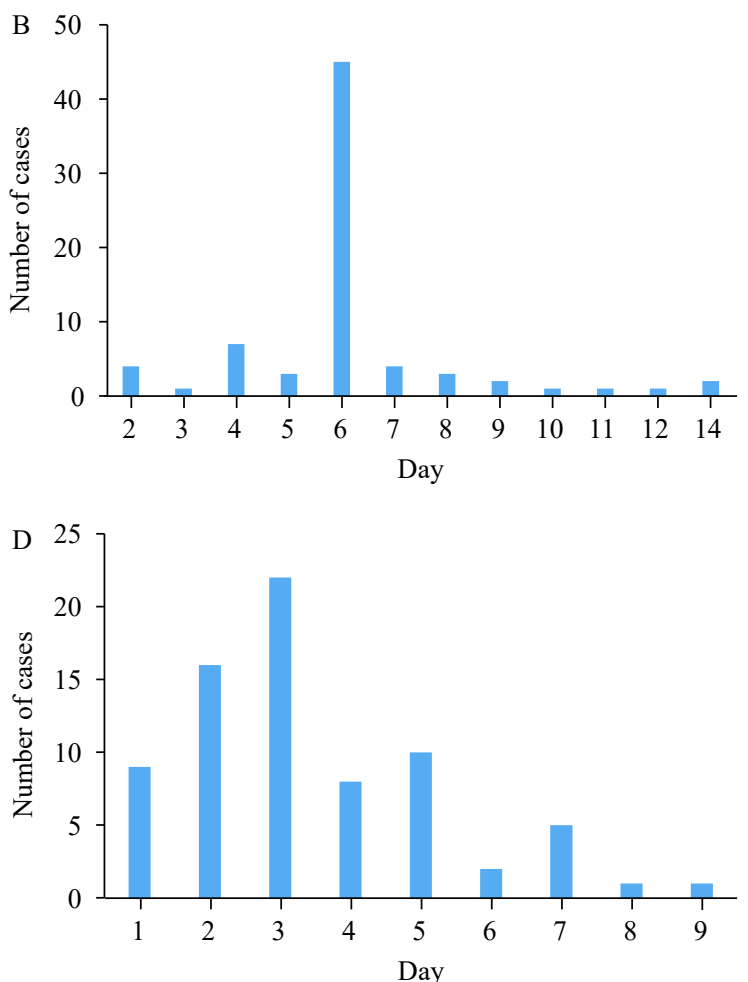

FIGURE 1. Observations during quarantine of the 74 first-generation COVID-19 cases infected by Patient $A$ on either January 10 or January 11, 2021, Jilin Province, China. (A) Age distribution. (B) Period between exposure to Patient A and turning PCR positive. (C) Period between exposure to Patient A and being confirmed as a COVID-19 case. (D) Period between turning PCR-positive and being confirmed as a COVID-19 case. 


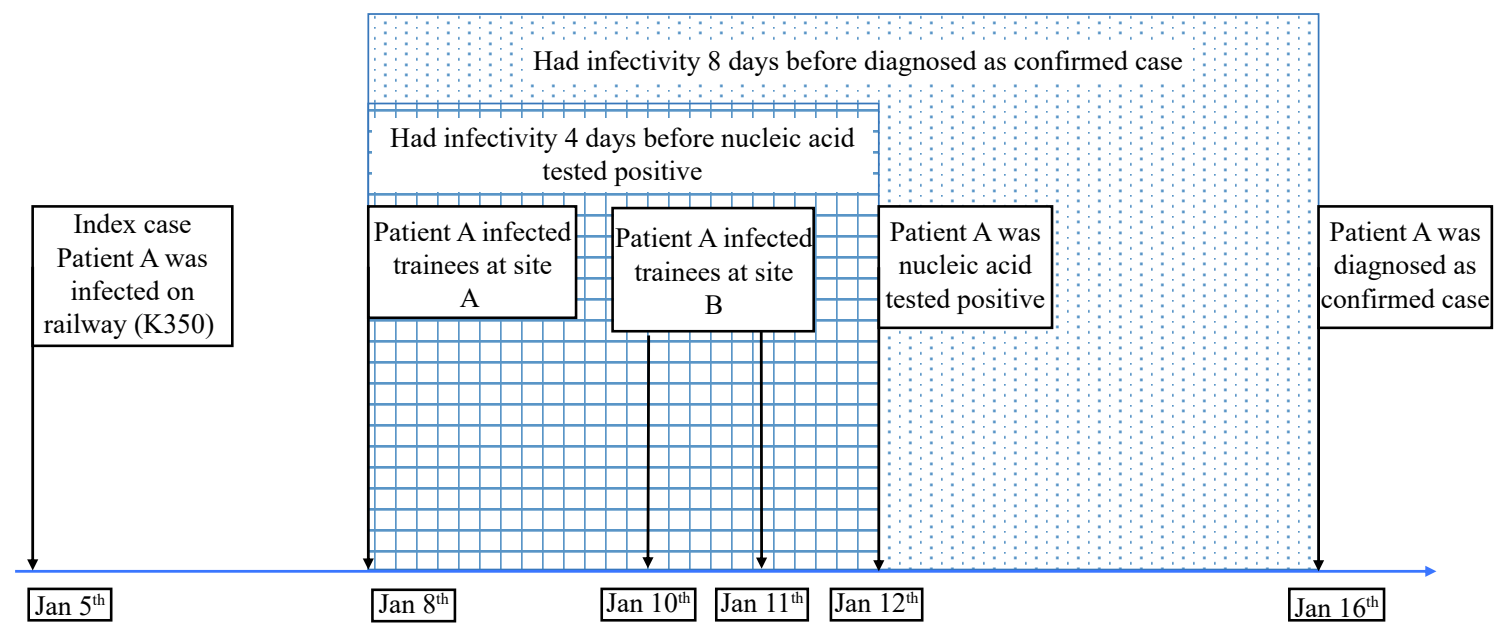

FIGURE 2. Timeline of infectivity of Patient A before and after testing positive with PCR and being confirmed as a COVID-19 case, January, 2021, Jilin Province, China.

Tonghua outbreak (6). By the time of the Tonghua outbreak, samples from close contacts of cases could be tested, and laboratory results obtained within 24 hours. Higher PCR throughput helped to efficiently find infected individuals among close contacts in order to quarantine contacts and stop chains of transmission. Based on the case finding with population-wide PCR testing used in this outbreak, we suggest that during an outbreak response, close contacts should be traced from PCR-positive individuals and suspected cases, rather than from suspected cases only.

Patient A caused a large cluster of COVID-19 cases infected during his lectures. Many cases were serious $-39 \%$ of cases were classified as critical or severe. All 74 of the first-generation cases developed symptoms, possibly indicating that Patient A was at an early stage of illness and secreting large amounts of virus (G). His lectures were lengthy and were held in small, nonventilated spaces, possibly increasing inhalation of virus. One recent study found viral loads among index cases to be a leading determinant of SARS-CoV-2 transmission. In that study, risk of transmission from symptomatic cases and shorter incubation times were strongly associated with viral loads during contact, in a dose-dependent manner (8).

Local CDC staff traced his close contacts once Patient A tested positive by PCR on January 12. The close contacts were quarantined and tested with PCR daily. Once PCR-positive, they were transferred to fixed point medical institutions for medical observation and treatment, when indicated. If a PCR-positive case developed symptoms, clinicians would diagnose him or her as a confirmed COVID-19 case. All times were well documented, allowing precise analysis. We measured the incubation periods of the confirmed cases and found the average to be 8.64 days. We found that the time between turning PCR positive and developing symptoms was 2.49 days.

Patient A's infection timeline was clear: he was infected on January 5; gave lectures and infected participants on January 8,10 , and 11 ; turned PCRpositive on January 12; and diagnosed as a confirmed COVID-19 case on January 16. The clear timeline enabled determination of infectivity before diagnosis. Patient A was infectious at least eight days before being diagnosed as a confirmed case and was infectious at least four days before turning PCR-positive. This timing implies that using two days between close contact and PCR positivity as the risk period for infection is too short of a time interval as many infections would be missed. One report in China found transmission as early as five days before onset of symptoms in an index case (9). Our investigation suggests that the close contact tracing period should be four days before samples are obtained for PCR testing of asymptomatic infected persons, and even longer for confirmed cases with symptoms.

This report was subject to at least two limitations. First, all subjects were elderly; the corresponding time periods and degrees of infectivity may be different for younger people. Second, all subjects were firstgeneration cases; subsequent generation cases may differ by infectivity or timing. However, studying firstgeneration cases while under quarantine or medical observation allowed precise timing of infection or illness events.

(Data updated on February 1, 2021)

Acknowledgements: Shicheng Yu, Xudong Li, and 


\section{Lance Rodewald from China CDC Weekly. Funding: Beijing Natural Science Foundation (L202008).}

doi: $10.46234 / \mathrm{ccdcw} 2021.093$

\# Corresponding authors: Dapeng Yin, yindp@chinacdc.cn; Laishun Yao,yaolaishun@126.com.

\footnotetext{
${ }^{1}$ Tonghua Center for Disease Control and Prevention, Tonghua, Jilin, China; ${ }^{2}$ Chinese Center for Disease Control and Prevention, Beijing, China; ${ }^{3}$ Zhejiang Center for Disease Control and Prevention, Hangzhou, Zhejiang, China; ${ }^{4}$ Jilin Center for Disease Control and Prevention, Changchun, Jilin, China.

\& Joint first authors.
}

Submitted: February 22, 2021; Accepted: March 23, 2021

\section{REFERENCES}

1. World Health Organization. COVID-19 strategy update - 14 April 2020. Geneva: WHO; 2020. https://www.who.int/publications/i/item/ covid-19-strategy-update---14-April-2020. [2021-2-21].

2. World Health Organization. Critical preparedness, readiness and response actions for COVID-19: interim guidance. Geneva: WHO; 2020. https://www.who.int/publications-detail/critical-preparedness- readiness-and-response-actions-for-covid-19. [2021-2-22].

3. World Health Organization. Contact tracing in the context of COVID19: interim guidance. Geneva: WHO; 2020. https://www.who.int/ emergencies/diseases/novel-coronavirus-2019. [2021-2-21].

4. Chinese Center for Disease Control and Prevention. Guidelines for investigation and management of close contacts of COVID-19 cases. China CDC Wkly 2020;2(19):329 - 31. http://dx.doi.org/10.46234/ ccdcw2020.084.

5. The Novel Coronavirus Pneumonia Emergency Response Epidemiology Team. The epidemiological characteristics of an outbreak of 2019 novel coronavirus diseases (COVID-19) - China. China CDC Wkly, 2020;2(8):113-22. http://dx.doi.org/10.46234/ccdcw2020.032.

6. Yao LS, Luo MY, Jia TW, Zhang WG, Hou ZL, Gao F, et al. COVID19 super spreading event amongst elderly individuals - Jilin Province, China, January 2021. China CDC Wkly 2021;3(10):211 - 3. http://dx. doi.org/10.46234/ccdcw2021.050.

7. Zhou L, Yao LS, Hao P, Li C, Zhao QL, Dong HL, et al. COVID-19 cases spread through the K350 train - Jilin and Heilongjiang Provinces, China, January 2021. China CDC Wkly 2021;3(8):162 - 4. http://dx. doi.org/10.46234/ccdcw2021.026.

8. Marks M, Millat-Martinez P, Ouchi D, Roberts CH, Alemany A, Corbacho-Monné M, et al. Transmission of COVID-19 in 282 clusters in Catalonia, Spain: a cohort study. Lancet Infect Dis 2021;21(5):62936. https://doi.org/10.1016/S1473-3099(20)30985-3.

9. Zhang Y, Muscatello D, Tian Y, Chen YW, Li S, Duan W, et al. Role of presymptomatic transmission of COVID-19: evidence from Beijing, China. J Epidemiol Community Health 2020;75(1):84 - 7. http://dx. doi.org/10.1136/jech-2020-214635. 